\title{
100 Successful Consecutive Peroral Endoscopic Myotomies (POEMs) for Long-Term Treatment of Esophageal Achalasia Including Complex Achalasia Patients. The Greek Experience
}

\section{Nikolas Eleftheriadis ${ }^{1-4}$, Haruhiro Inoue ${ }^{3}$, Haruo Ikeda ${ }^{3}$, Manabu Onimaru ${ }^{3}$, Nikolas Evgenides ${ }^{2}$, Ioanna Bravita ${ }^{2}$, Andreas Protopapas ${ }^{4}$, Eleni Eleftheriadou ${ }^{2}$, Grace Santi ${ }^{3}$, Theodora Kali ${ }^{5}$ and John Katsogridakis $^{1}$}

${ }^{1}$ Metropolitan Hospital Athens, Greece

${ }^{2}$ Euromedica Kyanous Stauros, Thessaloniki, Greece

${ }^{3}$ Digestive Diseases Center, Showa University, Koto Toyosu Hospital, Japan

${ }^{4} 1^{\text {st }}$ Propedeutic Department of Internal Medicine, Endoscopy Department, AHEPA

University Hospital, Greece

${ }^{5}$ Gastroenterology Unit, Larnaka General Hospital, Cyprus

*Corresponding Author: Nikolas Eleftheriadis, Gastroenterologist, Euromedica

Kyanous Stauros, Thessaloniki, Greece.
Received: December 16, 2019

Published: February 11, 2020

(C) All rights are reserved by Nikolas

Eleftheriadis., et al.

DOI: 10.31080/ASGIS.2020.03.0118

\begin{abstract}
Introduction: Per Oral endoscopic myotomy (POEM) has been accepted as minimal-invasive, endoscopic treatment option for longterm treatment of all types and stages of esophageal achalasia. The aim of the present study is to report our experience from 100 successful consecutive POEMs in Greek patients including complex achalasia patients.

Patients and Methods: We report on 100 consecutive patients with manometricaly proved esophageal achalasia (26 patients type I, 41 type II, 33 type III, and 21 with sigmoid esophagus), age range 17-92-years, mean age 55-years, 59 males, successfully treated by POEM from 2013-2019. Eckardt score was 7-12 (type III). POEMs were performed under general anesthesia with intubation, according to previous description. During POEM $\mathrm{CO}_{2}$ insufflation was obligatory used, while the TT-J knife was the main knife used in all POEM procedures and in all POEM stages. Eckardt score, esophagogram and manometry before and after POEM was used for evaluation of our results. POEM time was 45-130 mins. Follow-up was one month to six years.

Results: Selective circular myotomy 10-22 cm in length was successfully completed in all patients, without severe acute or late complications. Twelve (12\%) showed moderate pneumomediastinum and pneumoperitoneum, which was successfully managed by abdominal needle drainage during procedure. Five patients, during our first 20 POEMs, had severe intratunnel bleeding, which was successfully controled by coaggrasper, two patients had small mucosal injuries immediately recognised and successfully closed by clips and two patients showed mild pleural collection treated conservatively. Finally outcome was uneventful without any further clinical concequences. No other short or long-term serious complications were reported. Patients were discharged after one to three days hospitalization. During the follow-up one month to six years after POEM procedure all patients are alive, the majority (90\%) had complete clinical improvement of their severe symptoms, while five patients successfully treated by redo- POEM in short term, which finally solved the problem, while in another five patients with complete dysphagia ( 3 with failed Heller, 2 octogenarians with sigmoid megaesophagus), POEM resulted in moderate improvement of dysphagia, however sufficient to maintain adequate nutrition for five years post-POEM. Erosive esophagitis was reported in 7\%.

Conclusions: Our results are in accordance to international data and proved the safety and efficacy of POEM as long-term treatment of all types of achalasia, including complex achalasia patients, end-stage sigmoid type achalasia and failure of all other previous therapies and failed Heller. In all these cases, POEM was the only realistic therapeutic option, gave solution in long-term, where all other treatments failed or contra-indicated.
\end{abstract}

Keywords: Achalasia, Heller Myotomy; Per-Oral Endoscopic Myotomy (POEM); Complex Achalasia; Submucosal Endoscopy; HRTM

\section{Introduction}

PerOral Endoscopic Myotomy (POEM) [1] has been accepted as minimal invasive, long-term endoscopic treatment option for all types of esophageal achalasia (according to Chicago [2] classification)], but also of other esophageal motility disorders [3]. In specialized centers the POEM technique has been extended and applied for sigmoid type achalasia [4,5], in failure of Heller (LHM) myotomy [6,7], and also in failure of previous POEM [4].

Although POEM has been already established as an accepted treatment option for achalasia [8-10], little experience exist for the feasibility, safety and efficacy of POEM for complex achalasia cases 
$[11,12]$. The aim of the present study is to report our experience from 100 successful consecutive POEMs in Greek patients including complex achalasia patients.

\section{Patients and Methods}

We report the safe and successful application of the POEM procedure in 100 consecutive Greek patients with manometricaly proved esophageal achalasia, age range 17- to 92-years-old, mean age 55-years, 59 males, 38 of them treated in Endoscopy Department, from 2013-2019. According to Chicago 2 classification, 26 patients were classified as type I, 41 patients as type II, 33 patients as type III, 21 patients had sigmoid esophagus and 12 patients with failed Heller. Eckard score was 7-12 (type III).

All POEMs procedures of this study were performed, by 3 operators ( $\mathrm{Hi}, \mathrm{NE}, \mathrm{JK}$ ), under general anesthesia with intubation of the patients in the supine position, either in the endoscopy department or in operation room, according to previous description $[1,13]$. The operator's learning curve was 10 POEMs. A standard forward-viewing diagnostic gastroscope was used for all POEMs, with a transparent distal small "taper" cap (ST Hood (Fujicup) attached and securely fixed at the tip of the endoscope. An oblique cap (MH-588 (OLYMPUS) was used for clipping the esophageal opening at the end of POEMs. An over tube was used for stabilization of the endoscope and easy access to esophagus.

Carbon dioxide (CO2) gas insufflation was provided with the aid of a $\mathrm{CO} 2$ insufflator (UCR; Olympus). The air/water button was closed during POEM.

A triangle-tip knife (TT-Knife) (KD-640L; Olympus corp.) was used for submucosal dissection and myotomy in all POEMs and all steps of each POEM, while in 56 patients the new TT-J knife was used, with the capability to inject solution while cutting. A coagulating forceps (Coagrasper, FD-411QR; Olympus Corp) were used for hemostasis and coagulation of large vessels, when encountered during dissection.

High frequency electrosurgical energy generator $(\mathrm{V} / \mathrm{O}$ 300D ERBE; Tubingen, Germany) was used for submucosal dissection and myotomy. Settings were individually adjusted during the operation.

A normal saline $0.9 \%$ with indigo carmine dye $(0.3 \%)$ was used for submucosal injection. Indigo carmine was important for blue staining of the submucosal space. The initial injection was performed with a 25gauge injection needle, while subsequently submucosal injection was performed using the injection catheter. were flushed into the submucosal tunnel. For final closure of the mucosal entry site, different small or larger hemostatic clips were applied.

All patients were placed on a clear liquid diet for 24 hours before procedure and fasting from previous afternoon, admitted the morning of the operation and after of routine preoperative examinations, POEM was performed. Endoscopic clearance of esophageal contents was routinely performed before POEM, while in patients with sigmoid-type achalasia, endoscopic clearance from residues was performed on the day before POEM, to avoid aspiration during induction of anesthesia.

Prophylactic intravenous antibiotics, usually cefuroxime 750mg IV three times per day, was started during POEM and continued since discharge in uncomplicated cases. Then patients are transitioned to an additional four days of oral antibiotics. No other specific measures were followed.

POEM started with a mucosal incision, followed by submucosal tunnel creation crossing the esophagogastric junction (GEJ) and myotomy (Figures 1-2). Finally, the mucosal entry was closed with endoscopic clip placement. Eckardt score, esophagogram before and after POEM (Figure 3) and manometry, particularly high-resolution topographic manometry (HRTM) [4] was used for achalasia diagnosis and evaluation of efficacy of POEM. Follow-up was one month to six years.

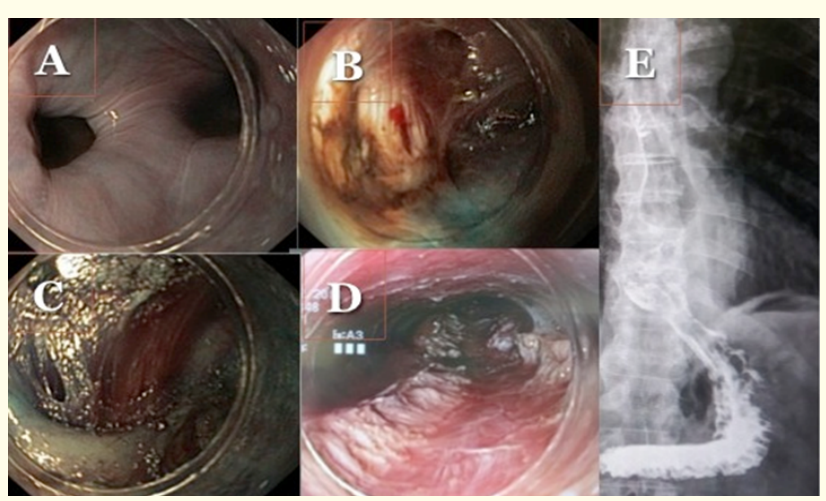

Figure 1: A-E. Successful POEM in 79-years-old male with spastic type III achalasia and multiple large esophageal diverticulums: A) Orifices of esophageal diverticulums in the middle esophagus from esophageal lumen during POEM. We recognize the blue dye. $\mathrm{B}, \mathrm{C}$ ) Submucosal tunnel. We recognize the thick muscle layer and the orifices of the diverticulums within the submucosal tunnel. D) Long myotomy length up to $22 \mathrm{~cm}$. E) Esophagogram the same afternoon after POEM, showed rapid passage of the contrast medium (gastrographin) to the stomach.

At the end of myotomy and before closure of the mucosal entry, $80 \mathrm{mg}$ Gentamycin diluted into $20 \mathrm{cc}$ normal saline solution (0.9\%) 


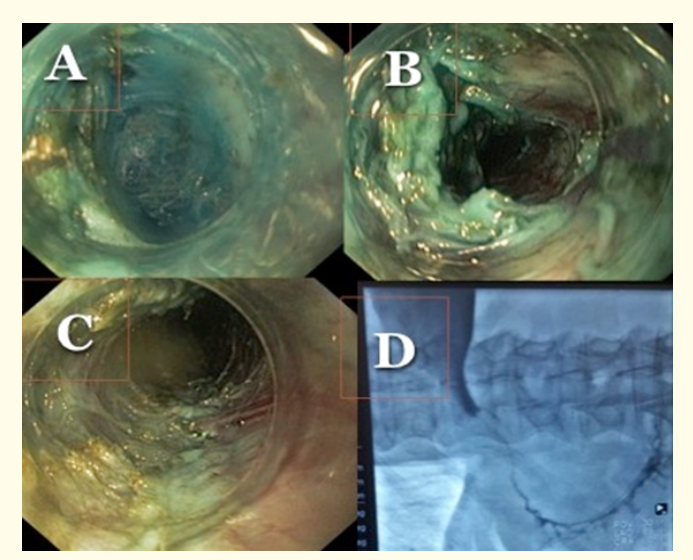

Figure 2: A-D. Successful POEM in 77-years-old male with spastic type III achalasia, sigmoid esophagus and severe submucosal fibrosis due to failed multiple Botox injections: (A, B) Submucosal tunnel. Identification of severe submucosal fibrosis. (C) Selective posterior circular myotomy is performed using TT-J knife. (D) Esophagogram the same afternoon after POEM, showed rapid passage of the contrast medium (gastrographin) to the stomach.

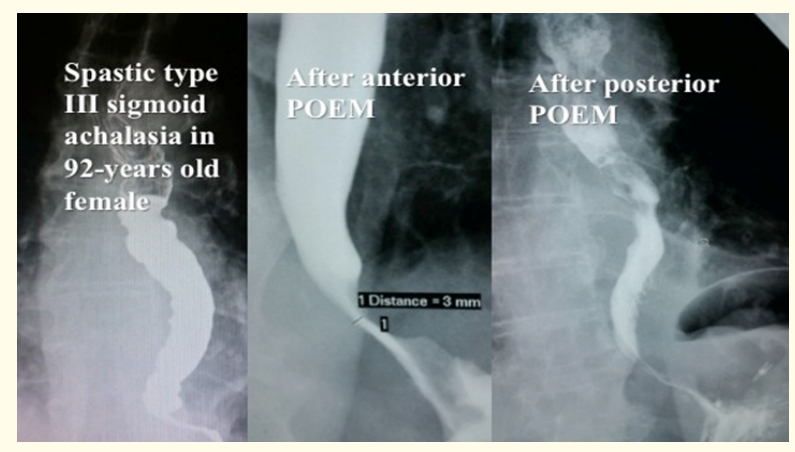

Figure 3: Esophagogram of 92-years-old female with spastic type III achalasia before and after anterior and redoposterior POEM within a month. Rapid passage of the contrast medium after second redo-POEM.

\section{Results}

All POEM procedures were successfully completed uneventfully, according to the international standards (feasibility 100\%) and myotomies were effectively extended $1-3 \mathrm{~cm}$ below the GEJ. The majority of the patients (90\%) discharged the next day after POEM.

POEM technique was also safely and effectively applied to 32 elderly patients, some of them octogenarians up to 92-years-old, with absolute contraindications for surgery, due to serious comorbidities such as ischemic cerebrovascular disease, severe coronary artery disease, cardiac arrhythmias, lymphoma under chemotherapy, etc. and failure of all other previous therapies. In all these patients, POEMs were performed under continuous anticoagulation treatment with low-molecular-weight-heparin (LMWH).
POEM was also successfully and effectively applied to 12 patients with failure of previous Heller myotomy. Particularly a 62-years-old female patient with persistent complete dysphagia after three previous failed Heller and gastropexy, underwent successfully POEM, which solved the problem in long-term up to two years follow-up post-POEM (Figure 4).

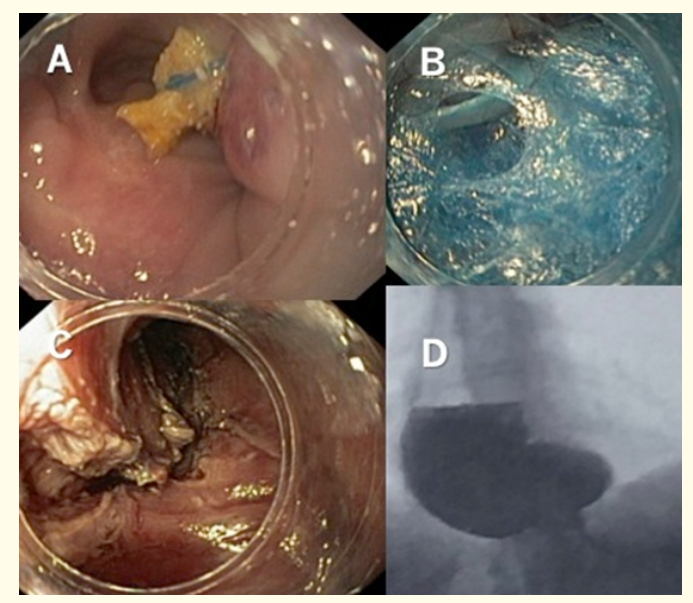

Figure 4: Successful POEM after three failed Heller in a 62-years-old female with sigmoid type II achalasia and megaesophagus. A) Dilated esophagus and suture from previous Heller and gastropexy. B) Submucosal tunnel and suture in the tummel.

C) Myotomy. D) Rapid passage of contrast medium after POEM.

Selective circular myotomy $10-22 \mathrm{~cm}$ in length was successfully completed in all patients with extension of myotomy 2-3 cm below the GEJ, as this was controled by identification of blue dye at the gastric cardia at the end of the procedure. We performed 10 anterior and 90 posterior POEMs according to the particularities of our cases. As the majority of our cases were complex achalasia cases, or failure of previous anterior surgical myotomies or failed anterior POEMs, or sigmoid megaesophageal cases, we considered the posterior approach as our first choice. POEM time was 45-130 mins, with procedure time reduced to less than one hour for the last 65 consecutive POEMs. Patients normally started drinking the same afternoon or the following day after POEM, discharged 1-3 days after and returned to their normal activities immediatelly during the first week after POEM. No short or long term serious side effects were reported and all patients are alive up to six years after POEM.

Twelve patients (12\%) showed moderate pneumomediastinum and pneumoperitoneum, which was successfully managed by abdominal needle drainage during procedure, without any further clinical impact. Actually pneumoperitoneum or pneumomediastinum should not be considered as complications but as normal concequence of POEM, in accordance to pneumomediastinum and pneumoperioneum in thoracoscopic or laparoscopic procedures respectively. 
Two patients showed mild asymptomatic pleural collection which resolved in a few days after POEM, without any further clinical significance, and in one patient the clip-endoloop technique was successfully used to close the mucosal entry.

In five patients during our first 20 POEMs severe intratunnel bleeding was appeared which was finally successfully controled by coaggrasper. POEMs were successfully completed theirafter. Two of these patients trasfused for safety reasons, without any further clinical consequences.

Small mucosal injuries during POEM reported in two patients, which were immediately recognised and successfully managed either with change the direction of submucosal tunnel and performing myotomy far from injury site or with succesfull closing using clips. No further clinical consequences were reported in these two cases. Outcome was uneventful with conservative measures without any further clinical concequences in all patients of this study.

During the six years follow-up after POEM mild erosive esophagitis stage I-II (LA classification) and mild GERD symptoms were reported in 7 patients (7\%) succesffully managed by PPIs on demand and modification of the life style. No long term serious GERD is reported to our case series. No other short or long-term complications related to POEM were reported and all our patients enjoy a satisfactory quality of life.

In five patients, two of them octogenarians, inial anterior POEM was failed to complete myotomy and second posterior POEM (redo-POEM) finaly solved the problem in long term. In a 92-years-old female with severe sigmoid type III achalasia, complete dysphagia and recurrent serious episodes of aspiration pneumonia, posterior redo-POEM was successfully and effectively performed within one month after initial failed anterior POEM. This particular patient had absolute contra-indications for surgery due to serious comorbidities, while all previous therapies failed. Four years later this particular female patient is totally perfect, eating and drinking normally and lives a normal life in Athens.

During follow-up one month to six years after POEM, the great majority of patients (90\%) showed total clinical improvement (Eckardt score 0-3 after POEM). Five patients underwent second redo-POEM (5\%), which finally solved the problem, while in five patients with complete dysphagia (3 patients with previous failed Heller, 2 with sigmoid megaesophagus with more than $10 \mathrm{~cm}$ esophageal dilatation), POEM resulted in moderate improvement of dysphagia (Eckardt score: 5 after POEM), however sufficient to maintain adequate nutrition for six years post-POEM.

\section{Discussion}

The results of the present study were comparable to international standards [14], in terms of feasibility, safety and efficacy of
POEM technique, while there was no restriction on the application of POEM regarding age, type or stage of achalasia.

POEM is performed during endoscopy, it is relatively painless and bloodless procedure, without scar and short hospitalization time (one day), while recovery time is also short, patients mobilized the same day, started drinking the same afternoon after POEM and returned to all their daily activities directly within a few days.

An interesting finding of our study is that the majority of our patients (74\%) were type II (41\%) or III (33\%) achalasia, that means longest myotomy length, up to $22 \mathrm{~cm}$ in our case series, was necessary to solve the dysphagia symptoms. The longest myotomy length achieved by POEM, is a major advantage of POEM, which is practically impossible with any other method.

Nevertheless, the oldest surgical Heller myotomy (1913), but also the more recent laparoscopic (LHM) myotomy, are so aggressive and invasive, because they require both thoracotomy and abdominal incision to approach the esophagus, that practically make them prohibitive in terms of risk. Moreover, in case of failure, redoHeller is practically impossible, while it increases the risk of complications, as it was the case in a 62-years-old female patient of our study, with three previous failed Heller and complications.

In contrast, POEM is not only a minimal invasive procedure, approximating the diseased circular esophageal muscle responsible for achalasia, directly and easily through the submucosal tunnel, permitting safe and efficient long myotomy, but has the advantage to be easily repeated (redo-POEM), also in short term, after initial failed POEM, as it was the case in five of our patients.

Another interesting finding of our study is that in the majority of our patients, POEM was successfully applied to complex achalasia cases without exceptions, such as in 32 elderly and octogenarian patients with serious comorbidities and absolute contraindications for surgery, in 21 end-stage sigmoid type achalasia patients with megaoesophagus up to $10 \mathrm{~cm}$, in patients with concomitant multiple large esophageal diverticulum and severe submucosal fibrotic cases and finally in failures of all other previous therapies (failed repeated Botox injections and ballon dilatations) and in 12 failed Heller patients, which supposed to be the ultimate solution for achalasia. In all these cases, POEM was not only a simply alternative therapy to other existing treatments, but to our opinion and according to our results, POEM is the only realistic therapeutic option in cases where all other treatments failed or contra-indicated.

Another important advantage of POEM, is that after POEM no anti-reflux procedure is necessary, because during POEM the normal anti reflux mechanisms (diafragmatic cure and angle of His) remain intact due to internal access through the submucosal tunnel, as it is also proved by the very low incidence of GERD disease, usu- 
ally mild and well controlled by PPIs on demand in our study (7\%), which is in accordance to international literature (10-20\%) [2].

In contrary, in surgical or LHM myotomy, the normal anti reflux mechanisms were disrupted due to access the esophageal muscle from outside and so anti reflux procedures are obligatory.

\section{Conclusion}

In conclusion, according to our successfull results and in view of exciting results in international level, we consider POEM safe and effective therapy of choice for all types of esophageal achalasia, including complex achalasia cases. Substantially POEM proved to be superior to all other therapies, including the invasive and pain full laparoscopic Heller myotomy, not only in terms of miniall invasivity, but mainly in terms of feasibility, safety and efficacy and in cases where all other treatments failed or contraidicated. POEM has been established worldwide and appears to have replaced all prior treatments for achalasia. Further experience and long-term follow-up in complex achalasia cases is necessary and awaited.

\section{Author Contributions}

Eleftheriadis N: Design research, performed research, Performed POEMS, Wrote the paper, analyzed the data

Inoue H: Design research, performed research, Performed POEMS, analyzed the data

Ikeda H: Performed research

Onimaru M: Performed research

Eugenides N: Performed research

Bravita I: Performed research

Protopapas A: Performed research, performed POEMs

Eleftheriadou E: Performed research

Grace Santi: Performed research

Dora Kali: Performed research

Katsogridakis J: Design research, performed research, Performed POEMS, analyzed the data.

\section{Conflict of Interest}

None.

\section{Disclosure}

All authors disclosed no financial relationships relevant to this publication.

\section{Bibliography}

1. Inoue H., et al. "Peroral endoscopic myotomy (POEM) for esophageal achalasia”. Endoscopy 42 (2010): 265-271.

2. Pandolfino JE., et al. "Achalasia: a new clinically relevant classification by high-resolution manometry". Gastroenterology 135 (2008): 1526-1533.

3. Shiwaku H., et al. "Successful treatment of diffuse esophageal spasm by peroral endoscopic myotomy". Gastrointestinal Endoscopy 77 (2013): 149-150.
4. Onimaru M., et al. "Greater curvature myotomy is a safe and effective modified technique in per-oral endoscopic myotomy (with videos)". Gastrointestinal Endoscopy (2015).

5. Eleftheriadis N., et al. "Successful peroral endoscopic myotomy for radical treatment of sigmoid-type esophageal achalasia by Greek gastroenterologists". Annuals of Gastroenterology 27 (2014): 430-431.

6. Zhou PH., et al. "Peroral endoscopic remyotomy for failed Heller myotomy: a prospective single-center study". Endoscopy 45 (2013): 161-166.

7. Onimaru M., et al. "Peroral endoscopic myotomy is a viable option for failed surgical esophagocardiomyotomy instead of redo surgical Heller myotomy: a single center prospective study". Journal of American College of Surgeons 217 (2013): 598-605.

8. Cappell MS., et al. "Updated Systematic Review of Achalasia, with a Focus on POEM Therapy". Digestive Diseases and Sciences (2019)

9. Kumbhari V., et al. "Peroral endoscopic myotomy (POEM) vs laparoscopic Heller myotomy (LHM) for the treatment of Type III achalasia in 75 patients: a multicenter comparative study". Endoscopy International Open 3 (2015): E195-201.

10. Costamagna G., et al. "Peroral endoscopic myotomy (POEM) for oesophageal achalasia: preliminary results in humans". Digestive Liver Disease 44 (2012): 827-832.

11. Bechara R., et al. "Peroral endoscopic myotomy (POEM) for complex achalasia and the POEM difficulty score". Digestive Endoscopy 31 (2019): 148-155.

12. Weche M., et al. "Revisional Procedures for Recurrent Symptoms After Heller Myotomy and Per-Oral Endoscopic Myotomy". Journal of Laparoendoscopic and Advanced Surgical Techniques $A$ (2019)

13. Eleftheriadis N., et al. "Training in peroral endoscopic myotomy (POEM) for esophageal achalasia". Therapeutics and Clinical Risk Management 8 (2012): 329-342.

14. Stavropoulos SN., et al. "The International Per Oral Endoscopic Myotomy Survey (IPOEMS): a snapshot of the global POEM experience". Surgical Endoscopy 27 (2013): 3322-3338.

\section{Assets from publication with us}

- Prompt Acknowledgement after receiving the article

- Thorough Double blinded peer review

- Rapid Publication

- Issue of Publication Certificate

- High visibility of your Published work

Website: www.actascientific.com

Submit Article: www.actascientific.com/submission.php Email us: editor@actascientific.com

Contact us: +919182824667 